\title{
Krankenvorstellung in der Moskauer dermatologischen Klinik
}

\author{
am 10./XII. - 27./XI. 1911.
}

1. Bogrow zeigt eine 35jähr. Frau mit Atrophia cutis maculosa. Seit $1 \frac{1}{2}$ Jahren rezidivierendes Jucken und Rötung zirkumskripter symmetrischer Stellen an Händen, Ellbogen, Knien, Trochant. maj., Füßen, wo eine leichte Atrophie mit starker Pigmentierung zu sehen ist. Keine Sensibilitätsstörungen. Schwache Pirquetreaktion. Zufällig wurde Rötung durch Antipyringaben erzeugt. prüfen.

Tschernogubow möchte die Diagnose durch Tuberkulininjektion

Mestscherski verteidigt die Diagnose der Atroph. cutis idiopathica maculosa.

Fo k in findet Ahnlichkeit mit Le pra maculosa.

Bogr ow hat keine Leprabazillen finden können. Wahrscheinlich handelt es sich um toxische Veränderung unbekannten Ursprungs.

2. Grintschar bespricht auf Grund zweier demonstrierter, mit Frfolg behandelter Lup. erythematodes-Fälle die Vorteile der Kohlensäureschneebehandlung: Von 38 Fällen von Lup. erytb, sind 20 geheilt. Bester Erfolg bei 9 Fällen von Acne rosacea.

Brytschew spricht sich anch für die Methode aus.

Bogrow sieht eine wichtige Bedingung der Wirkung im Dracke und in der Länge der Applikation.

Grintschar individualisiert diese Faktoren. Behandlungsdauer bei Acne rosacea 25--35 Sekunden.

3. Muchin stellt eine 40jährige Bäuerin mit Cancer mammae en cuirasse vor, bei welcher skleremartige Härte beider Briiste mit Retraktion der Papillae und Hautmetastasen in Form von sklerodermieartigen Plaques sich entwickelt hatte. Elephantiasis und Ödema der rechten oberen Extremität (Druck der Metastasen in der Axilla ?), Vergrößerung und Härte der Leber. Ilkterus. Mikroskopis ch: Cancer fibrosus atrophicans. Krankheitsdauer 1 Jahr 8 Monate.

Arch. f. Dermat. n, Syph. Bd. CXII. 
146 Krankenvorstellung in der Moskauer dermatol. Klinik.

4. Muchin demonstriert eine 66jährige Pfründnerin mit Pemphigus vulgaris und Psorias is vulgaris, welch beide gleichzeitig vor 5 Monaten aufgetreten sind. Keine Eosinophilie $(4 \%)$.

Bremener fragt über bakteriologische Untersuchung der Blasen. Machin fand sie steril.

5. Bogrow führt ein 8jähriges Mädchen mit Mikros porie vor. In Rußland sind die Fälle selten. Hauptsächlich findet man Mikrosporon lanosum.

6. Preobraschenski stellt einen 38jährigen Mann mit Dermatitis herpetiformis Dühringi vor. Keine Eosinophilie (3\%). Erfolg von Arseninjektionen und von 1\% Salizylpaste.

Mestscherski und Bagrow behaupten auf Grund klinischer Beobachtungen den toxischen Ursprung der Blasenaffektionen.

Priv.-Doz. S. Bogrow (Moskau). 